\title{
A COMPACTNESS RESULT FOR GENERALIZED RADON TRANSFORMS
}

\author{
Patrick Gérard, François Golse, and Bernt Wennberg
}

Let $X$ and $Y$ be two $C^{1}$ manifolds with $\operatorname{dim} X=m$ and $\operatorname{dim} Y=n$. Let $Z$ be a $C^{1}$ submanifold of the product $X \times Y$ with $\operatorname{codim} Z=l<\inf (m, n)$. It will be assumed that the restrictions to $Z$ of the two canonical projections associated to the product $X \times Y$ are submersions; they are denoted by $p_{1}$ and $p_{2}$ as follows:

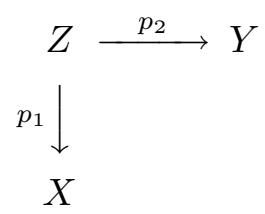

In the sequel, the fiber of $p_{1}$ above $x \in X$ is denoted by $Z_{x}^{\prime}$ and that of $p_{2}$ above $y \in Y$ by $Z_{y}^{\prime \prime}$. Let $\kappa \in C_{c}^{0}(|\wedge T Z|)$ be a compactly supported continuous density on $Z$; we shall denote $\mu=p_{1 *} \kappa$, the push-forward of $\kappa$ under $p_{1}$.

To the double submersion (1) is associated the generalized Radon transform

$$
K: C^{0}(Y) \mapsto C^{0}(X), \quad f \mapsto g \text { such that } p_{1 *}\left(\left(p_{2}^{*} f\right) \kappa\right)=g \mu .
$$

Such operators have been considered for example in [G-S, Ph-St1-2-3, So$\mathrm{St}]$; how they are related to the classical Radon transform is explained in [G-S, pp. 336-337, He]. We recall that, in the notations above, $p_{2}^{*} f=f \circ p_{2}$ is the pull-back of $f$ by $p_{2}$; for a density $\alpha$ on $Z$ whose restriction to each fiber $Z_{x}^{\prime}$ of $p_{1}$ is compactly supported, the push-forward $p_{1 *} \alpha$ is the density on $X$ obtained by integrating $\alpha$ on each fiber $Z_{x}^{\prime}$ of $p_{1}$. For example, in the case where $Z$ has codimension $l=1$ in $X \times Y=\mathbb{R}^{n} \times \mathbb{R}^{n}$ and is defined by a $C^{1}$ scalar equation $\Phi(x, y)=0$, the operator $K$ can be put in the form

$$
(K f)(x)=\lim _{\epsilon \rightarrow 0^{+}} \frac{1}{\epsilon} \int_{|\Phi(x, y)| \leq \epsilon} f(y) a(x, y) d y
$$

Received March 11, 1996. 
for some $C^{0}$ function $a$. For the general case, we refer the reader unfamiliar with the notations used in (2) to formulas (10) and (17) which give the expression of $K$ in local coordinates.

The smoothing effect of these operators has been studied for example in [G-S, So-St, Ph-St2-3]; these papers appeal to the theory of Fourier integral operators and assume that all the objects $X, Y, Z, \mu$ and $\nu$ are $C^{\infty}$.

a] Assume that both projections $\pi_{1}$ and $\pi_{2}$ of $T^{*}(X \times Y) \sim T^{*} X \times T^{*} Y$ map bijectively $N^{*} Z \backslash\{0\} \rightarrow T^{*} X \backslash\{0\}$ and $N^{*} Z \backslash\{0\} \rightarrow T^{*} Y \backslash\{0\}$. Then $m=n$ and, as explained in [G-S, pp. 364-365], the operator $K$ is an elliptic Fourier integral operator of order $(l-n) / 2$ associated to the homogeneous canonical relation $N^{*} Z$ (which is the graph of the canonical transform $\left.\pi_{2} \circ\left(\pi_{1 \mid T^{*} X \backslash\{0\}}\right)^{-1}\right)$; hence $K$ extends as a continuous $\operatorname{map} L_{c o m p}^{2}(Y) \rightarrow H^{(n-l) / 2}(X)$.

b] The same result is proved in [So-St] in the case where $m \geq n \geq 2, l=1$ and $Z$ is defined by a $C^{\infty}$ equation $\Phi(x, y)=0$, under the assumption that the matrix

$$
\left(\begin{array}{cc}
0 & \partial \Phi / \partial x_{i} \\
\partial \Phi / \partial y_{j} & \partial^{2} \Phi / \partial x_{i} \partial y_{j}
\end{array}\right)
$$

has maximal rank on $Z$. In the case where the matrix (3) has a square submatrix of maximal dimension whose determinant vanishes at infinite order nowhere on $Z$, Sogge and Stein show the existence of $\left.p_{0} \in\right] 1, \infty[$ such that $K$ extends as a continuous map $L_{\text {comp }}^{p}(Y) \rightarrow W^{(n-l) / p, p}(X)$ for all $p \in] p_{0}, \infty[$ : see [So-St].

c] In [Ph-St2-3], the same type of problem is studied in the case where $n=m$ and $l=n-1$; the gain of regularity in the Sobolev scale of spaces is related to the vanishing order of the system of equations defining $Z$. For example, when $n=m=2, \Phi(x, y)=x_{2}-x_{1}-S\left(x_{2}, y_{2}\right)$ with $S(x, y)=a_{j} x^{j} y^{n-j}+\ldots+a_{k} x^{k} y^{n-k}, 1 \leq j<k \leq n-1, K$ extends to a continuous map $L_{\text {comp }}^{p}\left(\mathbb{R}^{2}\right) \rightarrow W^{1 / n, p}\left(\mathbb{R}^{2}\right)$ provided that $n / k<p<n / j$, and to a continuous map $L_{\text {comp }}^{2}\left(\mathbb{R}^{2}\right) \rightarrow H^{1 / n}\left(\mathbb{R}^{2}\right)$ provided that $j=n / 2$ or $k=n / 2$.

In the present note, we give a geometric criterion on the double fibration (1) which is equivalent to the compactness of the operator $K$ in any $L^{p}$ space, $1<p<\infty$. This condition obviously encompasses all the situations described in a], b] and c] above and our result applies to much less regular objects $\left(C^{1}\right.$ instead of $C^{\infty}$ ) because we do not use Fourier integral operators. Instead, we use a compactness result on averages of solutions of PDEs (theorem 2.5 of [Gé1]) which generalizes the Velocity Averaging lemma established for kinetic models ([Ag], [GLPS]). The relevance of the notion of generalized Radon transforms to establish a crucial compactness 
property in the Boltzmann equation was recognized first by P.-L. Lions [L]. Indeed, the 'gain' part of the Boltzmann collision integral is an average over the surface of constant energy and momentum. Applications of the notion of Radon transform within this context can be found in [W] and [An].

Theorem. A necessary and sufficient condition for $K$ to extend as a compact operator

$$
K: L_{l o c}^{p}(Y) \mapsto L_{l o c}^{p}(X), \quad 1<p<\infty,
$$

is that

$$
\begin{aligned}
& \forall(x, \xi) \in S^{*} X, \\
& \left\{z \in Z_{x}^{\prime} \mid(\xi, 0) \in N_{z}^{*} Z\right\} \text { is of measure } 0 \text { as a subset of } Z_{x}^{\prime} .
\end{aligned}
$$

Proof. We first show that the operator (3) is continuous under the only assumption that (1) is a double fibration. Let $1 \leq p<\infty$. Let $U$ be an open set in $X$ and $\chi$ a $C^{0}$ function supported in $U$; one has to show that, for any relatively compact open set $V \subset Y$, there exists $C_{U V}>0$ such that, for all $f \in L^{p}(Y)$ supported in $V$

$$
\|\chi K f\|_{L^{p}(X)} \leq C_{U V}\|f\|_{L^{p}(Y)} .
$$

Using partitions of unity to localize the problem, one can reduce the problem to the case where:

- On $\bar{U}$ and $\bar{V}, C^{1}$ local coordinates denoted respectively by $x=$ $\left(x_{1}, \ldots, x_{m}\right)$ et $y=\left(y_{1}, \ldots, y_{n}\right)$ are defined; one denotes further $y^{\prime}:=\left(y_{1}, \ldots, y_{l}\right)$ and $y^{\prime \prime}:=\left(y_{l+1}, \ldots, y_{n}\right)$ one assumes that $V=V^{\prime} \times V^{\prime \prime}$ with $V^{\prime} \subset \mathbb{R}^{l}$ and $V^{\prime \prime} \subset \mathbb{R}^{n-l}$. Similarly we shall use the notations $x^{\prime}=\left(x_{1}, \ldots, x_{l}\right)$ and $x^{\prime \prime}=\left(x_{l+1}, \ldots, x_{m}\right)$. Since $p_{1}$ is a submersion, one also assumes $Z \cap \bar{U} \times \bar{V}$ to be defined by the (system of) equation(s)

$$
y^{\prime}:=\left(y_{1}, \ldots, y_{l}\right)=\phi\left(x, y^{\prime \prime}\right) .
$$

Moreover, for all $\left(x, y^{\prime \prime}\right) \in \bar{U} \times \overline{V^{\prime \prime}}$, one can assume

$$
D\left(x, y^{\prime \prime}\right):=\operatorname{det}\left(\frac{\partial \phi_{i}}{\partial x_{j}}\left(x, y^{\prime \prime}\right)\right)_{1 \leq i, j \leq l} \neq 0
$$

(this condition expresses locally the fact that $p_{2}$ is a submersion).

- On $Z \cap \bar{U} \times \bar{V}$, the density $\kappa$ is in the form $k\left(x, y^{\prime \prime}\right)|d x|\left|d y^{\prime \prime}\right|$.

Consider

$$
\psi: \ni\left(x, y^{\prime \prime}\right) \mapsto\left(x^{\prime \prime}, \phi\left(x, y^{\prime \prime}\right), y^{\prime \prime}\right)
$$


which, by condition (7), defines a $C^{1}$-diffeomorphism $U \times V^{\prime \prime} \rightarrow \psi\left(U \times V^{\prime \prime}\right)$ (which is open in $\mathbb{R}^{m+n-l}$ ). Hence

$$
\chi\left(p_{2}^{*} f\right) \kappa=h|d x|\left|d y^{\prime \prime}\right|
$$

with

$$
h\left(x, y^{\prime \prime}\right)=f\left(\phi\left(x, y^{\prime \prime}\right), y^{\prime \prime}\right) \chi(x) k\left(x, y^{\prime \prime}\right) .
$$

Therefore

$$
\begin{aligned}
& \int_{U \times V^{\prime \prime}}\left|h\left(x, y^{\prime \prime}\right)\right|^{p} d x d y^{\prime \prime}= \\
& \int_{\psi\left(U \times V^{\prime \prime}\right)}\left|f\left(y^{\prime}, y^{\prime \prime}\right)\right|^{p}\left|\chi k\left(\psi^{-1}\left(x^{\prime \prime}, y^{\prime}, y^{\prime \prime}\right)\right)\right|^{p}\left|D\left(\psi^{-1}\left(x^{\prime \prime}, y^{\prime}, y^{\prime \prime}\right)\right)\right|^{-1} d x^{\prime \prime} d y^{\prime} d y^{\prime \prime} \\
& (11) \quad \leq C_{\chi} \sup _{\left(x, y^{\prime \prime}\right) \in U \times V^{\prime \prime}}\left(\left|\chi(x) k\left(x, y^{\prime \prime}\right)\right|^{p}\left|D\left(x, y^{\prime \prime}\right)\right|^{-1} \mid\right)\|f\|_{L^{p}(V)}^{p} .
\end{aligned}
$$

(where $C_{\chi}$ is a positive constant depending only on the support of $\chi$ ). In other words, the map

$$
L^{p}(V) \ni f \mapsto h \in L^{p}\left(U \times V^{\prime \prime}\right) \quad \text { where } h \text { is defined by (10) }
$$

is continuous, which implies the continuity of (3).

Next, we assume that the geometric condition (4) is satisfied and proceed to show that the operator $(3)$ is compact. It suffices to prove compactness in the case where $p=2$; the general case follows by Hölder's inequality. We localize again $x$ in $U$ and $y$ in $V$ (the general case follows from a classical argument involving partitions of unity).

Without loss of generality, we can assume that the function $k$ is of class $C^{1}$. Indeed, if $k$ is only continuous, one can pick a sequence $k_{n}$ of $C^{1}$ functions converging uniformly to $k$ on $\bar{U} \times \bar{V}^{\prime \prime}$ and the compactness follows from estimate (11) once it has been established in the case where $k$ is replaced with any of the $k_{n}$.

Let $\left(f_{\epsilon}\right)$ be a bounded family of $L^{2}(Y)$ supported in $V$ and let $\left(h_{\epsilon}\right)$ be the bounded family of $L^{2}\left(U \times V^{\prime \prime}\right)$ associated to $\left(f_{\epsilon}\right)$ by $(12)$. If $L\left(x, y^{\prime}, \partial_{x}\right)$ is a differential operator of the form

$$
L\left(x, y^{\prime \prime}, \partial_{x}\right)=\sum_{1 \leq i \leq m} a_{i}\left(x, y^{\prime \prime}\right) \partial_{x_{i}}
$$

such that $L \phi_{r}=0$ on $U \times V, \quad 1 \leq r \leq l$ 
then

$$
\left(L\left(x, y^{\prime \prime}, \partial_{x}\right) h_{\epsilon}\right)\left(x, y^{\prime \prime}\right)=f_{\epsilon}\left(\phi\left(x, y^{\prime \prime}\right), y^{\prime \prime}\right) L\left(x, y^{\prime \prime}, \partial_{x}\right)\left(\chi(x) k\left(x, y^{\prime \prime}\right)\right) .
$$

Let $a_{i}^{j}\left(x, y^{\prime \prime}\right)(1 \leq i \leq m, 1 \leq j \leq m-l)$ be $C^{1}$ functions on $U \times V^{\prime \prime}$ such that

$$
L^{j}\left(x, y^{\prime \prime}, \partial_{x}\right)=\sum_{1 \leq i \leq m} a_{i}^{j}\left(x, y^{\prime \prime}\right) \partial_{x_{i}} \text { satisfies (13) for } 1 \leq j \leq m-l
$$

and

$$
\forall\left(x, y^{\prime \prime}\right) \in U \times V^{\prime \prime},
$$
the family $\left\{\left(a_{i}^{j}\left(x, y^{\prime \prime}\right)\right) 1 \leq i \leq m \mid 1 \leq j \leq m-l\right\}$ is free.

Formula (14) shows that, for all $1 \leq j \leq m-l$, the family $\left(L^{j} g_{\epsilon}\right)$ is bounded in $L^{2}\left(U \times V^{\prime \prime}\right)$. To show that the family

$$
g_{\epsilon}(x)=\int_{V^{\prime \prime}} h_{\epsilon}\left(x, y^{\prime \prime}\right) d y^{\prime \prime} \quad \text { is relatively compact in } L^{p}(X)
$$

we appeal to theorem 2.5 of [Gé1] which says that (17) holds provided that

$$
\begin{aligned}
& \forall(x, \xi) \in U \times \mathbb{R}^{n} \backslash\{0\}, \\
& \text { meas }\left(\left\{y^{\prime \prime} \in V^{\prime \prime} \mid \forall 1 \leq j \leq m-l, \sum_{1 \leq i \leq m} a_{i}^{j}\left(x, y^{\prime \prime}\right) \xi_{i}=0\right\}\right)=0 .
\end{aligned}
$$

According to (13) and (16), this is precisely equivalent to the condition

$$
\begin{aligned}
& \forall(x, \xi) \in U \times \mathbb{R}^{m} \backslash\{0\}, \\
& \text { meas }\left(\left\{y^{\prime \prime} \in V^{\prime \prime} \mid \xi \in \operatorname{span}\left\{\partial_{x} \phi_{r}\left(x, y^{\prime \prime}\right), 1 \leq r \leq l\right\}\right\}\right)=0 .
\end{aligned}
$$

According to (7) et (16), condition (18) clearly is equivalent to (4) since, if $z=\left(x, \phi\left(x, y^{\prime \prime}\right), y^{\prime \prime}\right)$, one has

$$
N_{z}^{*} Z \cap T_{x}^{*} X \oplus 0_{y}=\operatorname{span}\left\{\partial_{x} \phi_{r}\left(x, y^{\prime \prime}\right), 1 \leq r \leq l\right\} .
$$

This shows the compactness of (3) when (4) holds. Conversely, if the operator (3) is compact, (4) must hold because, as proved in [Gé2], (18) is a necessary and sufficient condition for the compactness (17).

By a standard interpolation argument involving the Dunford-Pettis characterization of weakly compact families in $L^{1}$ spaces, the Theorem above implies the following. 
Corollary. If condition (4) holds, the image by $K$ of any weakly relatively compact family of $L^{1}(Y)$ is compact in $L_{l o c}^{1}(X)$.

Remark 1. In some particular cases, the method used in the proof above can also give the optimal Sobolev regularity for (3). For example, consider the case where $n=m=2, l=1, X=\bar{U}$ and $Y=\bar{V}$ as in the proof above; suppose that $Z$ is given by the equation $y_{1}=A\left(y_{2}\right) x_{1}+B\left(y_{2}\right) x_{2}$. Then, the relevant operator $L$ in (15) is

$$
L\left(x_{1}, x_{2}, y_{2}, \partial_{x_{1}}, \partial_{x_{2}}\right)=B\left(y_{2}\right) \partial_{x_{1}}-A\left(y_{2}\right) \partial_{x_{2}} .
$$

According to [GLPS], $K$ extends as a continuous operator $L_{\text {comp }}^{2}(V) \rightarrow$ $H_{l o c}^{s}(U)$ with $0<s \leq 1 / 2$ if and only if

$$
\sup _{\left(\xi_{1}, \xi_{2}\right) \in S^{1}} \operatorname{meas}\left(\left\{y_{2} \in V^{\prime \prime}|| A\left(y_{2}\right) \xi_{1}+B\left(y_{2}\right) \xi_{2} \mid \leq \epsilon\right\}=O\left(\epsilon^{2 s}\right) .\right.
$$

The method in [GLPS] also shows that $K$ extends as a continuous operator $L_{\text {comp }}^{p}(V) \rightarrow H_{l o c}^{t}(U)$ when $(22)$ holds provided that $t<\inf (2 s / p, 1-2 s / p)$. Sharper regularity results in $L^{p}$ spaces have been obtained in [DPLM].

Although this type of result is less general than those obtained by Phong and Stein, the method of Velocity Averaging presented in [GLPS] gives in this case the optimal Sobolev regularity (in the $L^{2}$ case), whereas the method used by Phong and Stein, which is based on a Van der Corput type estimate (see [St, chapter VIII]), does not.

Remark 2. In the most general situation studied in the Theorem (with $C^{\infty}$ objects, though), one can obtain a sufficient condition for $K$ to extend as a continuous operator $L_{\text {comp }}^{2}(Y) \rightarrow H_{l o c}^{s}(X)$ for $0<s \leq(n-l) / 4$ : this condition is a uniform estimate for the measure of some tubular subset of the fiber $Z_{x}^{\prime}$ for all $x \in X$, in the style of (22). This follows from the results of [Gé3]. However, even if in some cases one can obtain in this way a better Sobolev regularity than in [Ph-St2-3], it is known that this regularity is not optimal. Indeed, when applied to the example (21)-(22), the result in [Gé3] would only give $H^{s / 2}$ regularity instead of $H^{s}$ under assumption (22).

\section{References}

[Ag] V. Agoshkov, Spaces of functions with differential difference characteristics and smoothness of solutions of the transport equation, Sov. Math. Dokl. 29 (1984), 662-666..

[An] H. Andreasson, A regularity property and strong $L^{1}$-convergence to equilibrium for the relativistic Boltzmann equation, to appear in SIAM J. of Math. Analysis.

[DPLM] R. DiPerna, P.-L. Lions, and Y. Meyer, $L^{p}$ regularity for velocity averages, Ann. Inst. Henri Poicaré 8 (1991), 271-287. 
[Gé1] P. Gérard, Microlocal defect measures, Comm. Partial Diff. Equations 16 (1991), 1761-1794.

[Gé2] Compacité par compensation et régularité 2-microlocale, Séminaire EDP 1988-89, Ecole Polytechnique, Paris.

[Gé3] _ Moyennisation et régularité 2-microlocale, Ann. Scient. Ecole Norm. Sup.

(1990), 89-121.

[GLPS] F. Golse, P.-L. Lions, B. Perthame, and R. Sentis, Regularity of the moments of the solution of a transport equation, J. Funct. Anal. 76 (1988), 110-125.

[G-S] V. Guillemin and S. Sternberg, "Geometric asymptotics", AMS, Providence, Math. Surveys Monographs 14 (1977).

[He] S. Helgason, "Geometric analysis on symmetric spaces", AMS, Providence, Math. Surveys Monographs 39 (1994).

[L] P.-L. Lions, Compactness in Boltzmann's equation via Fourier integral operators and applications I, J. Math. Kyoto Univ. 34 (1994), 391-427.

[Ph-St1] D. Phong and E. Stein, Hilbert integrals, singular integrals and radon transforms I, Acta Math. 157 (1989), 99-157.

[Ph-St2] _ Operator versions of the Van der Corput Lemma, Math. Res. Lett. 1 (1994), 27-34.

[Ph-St3] _ Models of degenerate Fourier integral operators and radon transforms, Ann. of Math. 140 (1994), 703-722.

[So-St] C. Sogge and E. Stein, Averages over hypersurfaces, smoothness of generalized radon transforms, J. Anal. Math. 54 (1990), 165-188.

[St] E. Stein, "Harmonic Analysis", Princeton University Press, 1994.

[W] B. Wennberg, Regularity in the Boltzmann equation and the radon transform, Comm. Partial Diff. Equations 19 (1994), 2057-2074.

Université Paris Xi and Ecole Normale Supérieure, D M i; Paris

E-mail address: gerard@dmi.ens.fr

Univerité Paris Vit and Ecole Normale Supérieure, D mi; Paris

E-mail address: golse@dmi.ens.fr

Chalmers University, G öteborg, Sweden

E-mail address: wennberg@math.chalmers.se 